\title{
Is the timing of infant cereal introduction a risk factor for
}

\section{celiac disease autoimmunity?}

Norris JM, Barriga K, Hoffenberg EJ, et al. Risk of celiac disease autoimmunity and timing of gluten introduction in the diet of infants at increased risk of disease. JAMA 2005:293:2343-51.

Background: Celiac disease, or glutensensitive enteropathy, has an estimated prevalence of $\mathrm{I} \%$ in North America and Western Europe. ${ }^{1}$ A diet of wheat gluten, rye and barley appears to trigger chronic inflammation in the small intestine. Typically, affected patients present in early childhood with abdominal cramps, diarrhea, malabsorption and nutrient deficiencies. In the past, parents often fed their children infant cereal, which contains gluten, from birth. Now, parents are advised to wait until children are 4-6 months of age before introducing cereal into their diet. More recently, the Canadian Pediatric Society has advocated breastfeeding only (i.e., no infant cereal) until the infant is 6 months of age. ${ }^{2}$ The link between when gluten is introduced into an infant's diet and the risk of celiac disease autoimmunity is unknown.

Design: This was a prospective observational study involving I560 children born in Denver, Colorado, who were at risk of type I diabetes or celiac disease on the basis of genetic tests for specific human leukocyte antigens (HLA-DR3 or DR4) of their cord blood or a family history of a first-degree relative with type I diabetes. Histories of the infants' diets were collected when the children were 3, 6, 9, I2 and I5 months of age. At each interview mothers were asked to report their child's intake of all types of milk, formula and foods over the last 3 months. Breastfeeding start and termination dates were also recorded. The children had blood tests for tissue transglutaminase (tTG) autoantibodies at 9, I5 and 24 months and yearly thereafter. Children were deemed to have celiac disease autoim- munity if 2 tGT autoantibody test results were positive or I tTG test and a small bowel biopsy were positive. Follow-up lasted for a mean of 4.8 years.

Results: Of the 1560 children, 5I developed celiac disease autoimmunity. Infants exposed to gluten in the first 3 months of life had a 5 -fold increased risk of autoimmunity compared with infants first exposed at 4-6 months (hazard ratio [HR] 5.17, 95\% confidence interval [CI] I.44-I8.57). Infants introduced to gluten at 7 months or later also had an increased risk of celiac disease autoimmunity compared with those exposed between 4 and 6 months (HR r.87, 95\% CI 0.97-3.60). The risk of autoimmunity was independent of the age of first exposure to rice and oats. Breastfeeding duration was similar for children who developed autoimmunity and those who did not.

Commentary: The study's strengths are its prospective nature, its frequent and relatively detailed dietary histories, and the number of children studied. Its limitations include possible recall biases, problems with generalizing the risk estimates to other populations, and possible problems with reliability and validity of the results. For example, although I56o children were studied, the primary outcome (celiac disease autoimmunity) was relatively rare. Only 5 I children in total developed celiac disease autoimmunity, and only 3 of the children exposed before 3 months of age developed it. Further, the development of celiac disease autoantibodies is only a proxy for the more clinically relevant outcome of celiac disease (only 25 children went on to have biopsy-proven disease).

Practice implications: The World Health Organization (WHO) recommends exclusive breastfeeding until 6 months of age to protect children from

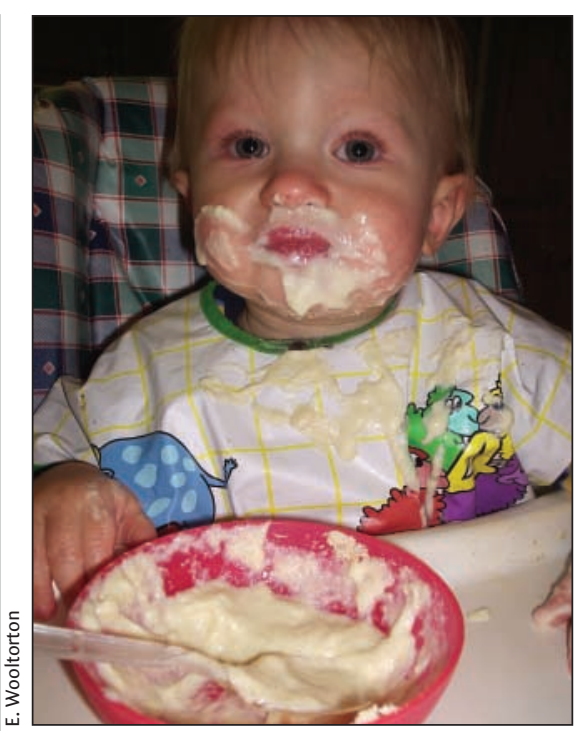

gastrointestinal infections, to prolong lactational amenorrhea and to increase postpartum maternal weight loss. The results of this study did not show that breastfeeding protected against celiac disease autoimmunity. However, at least for children predisposed to celiac disease or type I diabetes, avoiding cereal with wheat, rye or barley until a child is $4^{-6}$ months of age appears to reduce the risk of autoimmunity. Starting cereal before 3 months or after 7 months appears to increase the risk. Thus, although current recommendations in Canada and from the WHO are to breastfeed exclusively until at least 6 months of age, the results of this study suggest that it may be wise to consider introducing cereals at around the sixth month.

\section{Jeanette E. Belanger}

PGY 2 Resident

Department of Family Medicine

University of Ottawa

Ottawa, Ont.

Eric Wooltorton

CMAJ

\section{REFERENCES}

I. Farrell R. Infant gluten and celiac disease. Too early, too late, too much, too many questions. JAMA 2005;293:I9.

2. Boland M. Exclusive breastfeeding should continue to six months. Pediatrics and Child Health 2005;I0:I48. Available: www.cps.ca/english/statements/N/BreastfeedingMaro5.htm (accessed 2005 Oct 26). 